\title{
Breast cancer diagnosis in an early stage using novel deep learning with hybrid optimization technique
}

\author{
Kranti Kumar Dewangan ${ }^{1}$ (D) Deepak Kumar Dewangan ${ }^{1} \cdot$ Satya Prakash Sahu ${ }^{1}$. \\ Rekhram Janghel ${ }^{1}$
}

Received: 5 March 2021 / Revised: 17 January 2022 / Accepted: 21 January 2022 /

Published online: 25 February 2022

(C) The Author(s), under exclusive licence to Springer Science+Business Media, LLC, part of Springer Nature 2022

\begin{abstract}
Breast cancer is one of the primary causes of death that is occurred in females around the world. So, the recognition and categorization of initial phase breast cancer are necessary to help the patients to have suitable action. However, mammography images provide very low sensitivity and efficiency while detecting breast cancer. Moreover, Magnetic Resonance Imaging (MRI) provides high sensitivity than mammography for predicting breast cancer. In this research, a novel Back Propagation Boosting Recurrent Wienmed model (BPBRW) with Hybrid Krill Herd African Buffalo Optimization (HKH-ABO) mechanism is developed for detecting breast cancer in an earlier stage using breast MRI images. Initially, the MRI breast images are trained to the system, and an innovative Wienmed filter is established for preprocessing the MRI noisy image content. Moreover, the projected BPBRW with $\mathrm{HKH}-\mathrm{ABO}$ mechanism categorizes the breast cancer tumor as benign and malignant. Additionally, this model is simulated using Python, and the performance of the current research work is evaluated with prevailing works. Hence, the comparative graph shows that the current research model produces improved accuracy of $99.6 \%$ with a $0.12 \%$ lower error rate.
\end{abstract}

Keywords Breast cancer · Deep learning $\cdot$ Magnetic resonance imaging $\cdot$ Krill herd optimization Back propagation · African Buffalo optimization

Kranti Kumar Dewangan

kranti.d123@gmail.com

Deepak Kumar Dewangan

dkdewangan.phd2018.it@nitrr.ac.in

Satya Prakash Sahu

spsahu.it@nitrr.ac.in

Rekhram Janghel

rrjanghel.it@nitrr.ac.in

1 Department of Information Technology, National Institute of Technology, Raipur, Chhatisgarh 492010, India 


\section{Introduction}

The execution of a sophisticated system appliance in health service needs a more incorporated scheme [46] that involves information and communication technologies [20]. Smart data and data analysis with calculation is the expertise that makes great awareness in the field of the healthcare system [43]. In the contemporary era, the urbanized countries are leaving for the smart city's idea [6] in which all the needs and process is proscribed through a computer [34]. Moreover, one of the big challenges in smart cities is decision-making with all collected data, thus the current period is also called the data or information world [30]. In addition, health service is a huge issue in the realm [24] in that, the use of computers, smart devices, and smart systems are playing the main job in health service [21]. Furthermore, recognizing diseases at an early stage can decrease the hazard of human lives [41]. Moreover, one of the deadly diseases [17] globally is cancer that may generate in any region of the human body and might extend to other regions. Early detection of cancer in the benign phase is essential to protect one's life, and it needs to be prohibited from extending to other portions towards the malignant phase.

Several factors are upset a person's tendency; among that, breast cancer is the increasing disease of death for women. Hence, it is the main concern to minimize the death rate by early disease diagnosis. Also, breast cancer is described as the abandoned growth of anomalous cells in the milk-secreting glands in the breast. The finest effective method to diminish breast cancer death is an early diagnosis of the disease [19]. Also, the recognition of breast cancer requires a precise and consistent diagnostic formula that permits the physicians to differentiate gentle breast cancers from the evil ones deprived of straightly moving for operation biopsy. The idea of such detections is allotting the patients to any of the "benign" clusters or a "malignant" cluster [28]. A fine spine objective biopsy is a relatively easy and speedy method for cancer therapy for identifying growth. This method includes collecting a trial of cubicles or fluid from a nodule pursued by assessment [31] of the tissues under a microscope. If the knob cannot be sensed, the visualizing schemes are needed to identify the accurate site. In the ultrasound method, the doctor examines the spine on the ultrasound [15] and directs it towards the region.

Also, the mammograms are utilized at dissimilar angles, and through the aid of a PC, precise organs are shaped [1] though obtaining the prediction rate is difficult. Generally, deep learning methods are necessary for medical image processing, specifically in cancer recognition. They are exploited for identifying the breast tumors that the radiologist also suggests realizing the tumor treatment since they statically enhance the outcome of the expectations effectually. However, mammography images, Computed Tomography (CT) images, Positron emission tomography, and X-ray breast images are attained lower accuracy due to the falsenegative detection of cancer cells. Also, the MRI dataset is usually visualized using the t-SNE approach [37, 42]. In recent, the Fourier-Bessel series-based convolution Deep Learning (DL) approach has been effectively implemented to predict the COVID-19 diseases with the use of chest X-ray images [3] and electroencephalogram (EEG) signals [10]. In addition, the convolution DL methods such as squeezenet, GoogleNet, AlexNet, MobileNet-V2, VGG-16, ResNet-50 and ResNet-34, are utilized to predict the COVID symptoms in earlier stages [26]. Also, Fourier-Bessel series-based wavelet transform was introduced; the fundus images were used as input [4]. In another case, to find the non-focal and focal EEG signals 2D convolution neural model was executed for signal classification [22]. The current study is aimed to introduce an innovative deep learning model using MRI for detecting breast cancer in the former stage. Here, MRI is one of the highly suggested tests to diagnose and monitor breast cancer cells. Also, MRI can process the manner of soft tissue imaging. In addition, it provides 
accurate diagnostic results because it is not affected by dangerous radiation. Besides, the main motive of this proposed model is to reduce the death rate due to breast cancer. To reduce these issues already many techniques are implemented, but still, that technique has attained less accuracy because of noisy data. So, the proposed method utilized the MRI breast images for detecting and classifying breast cancer with the finest accuracy measure. Hence, the main goal of this research article is to improve the breast cancer classification accuracy and to predict cancer affection in an early stage with the help of cancer disease features and an effective preprocessing model. Here, the chief objective of this work is segmenting and classifying the breast tumor from the trained datasets.

The key contribution of this research is summarized as,

- Initially, the MRI Breast image dataset has been collected and trained to the machine

- Consequently, a novel BPBRW with HKH-ABO approach is developed to prognosis the breast cancer types in an initial stage.

- Here, an innovative Wienmed filter is introduced for performing the preprocessing process on the utilized dataset. In the preprocessing layer, the training noise were removed from the MRI data and error refined image data has been attained

- Moreover, the fitness of HKH-ABO function is utilized for classifying the disease types, which has enhanced the classification accuracy while predicting breast cancer

- Here, the breast cancer has been predicted in an earlier stage to reduce the death rate and treatment risks

- Finally, the performance metrics are validated with prevailing techniques in terms of accuracy, precision value, recall value, F1-score, and error rate.

The remaining arrangement of this work is prearranged as various sections that are, Section 2 investigates related works concerning breast cancer detection. Section 3 provides the problem definition, and Section 4 extends a detailed explanation of the introduced breast cancer recognition mechanism. Section 5 provides the investigational results and Section 6 completes the paper.

\section{Related works}

Some of the recent literature related to this current research is described below,

The MRI dataset for breast cancer is utilized to develop computer-aided frameworks by traditional or deep learning prototypes, but the large datasets are complicated to process in some cases. Benhammou et al. [2] proposed the taxonomy model that can classify the disease into four dissimilar reformulations to overwhelm this difficulty. In this approach, the public dataset is utilized to solve the issues that are called the BreakHis dataset, which makes the classification and prediction process easier. But, it attained lower prediction accuracy than other approaches.

Sadhukhan et al. [33] proposed the KNN and SVM models to detect breast cancer earlier because the recognition in the last stage causes death. Using this classifier and machine learning model, they can classify the cell nuclei and predict the beginning stage of tumor growth. After the prediction, the performance of the projected model with prevailing works is evaluated and hence achieved better results. However, this method takes more time to detect the tumor cells. 
The fuzzy clustering model is a trending model in image processing that is employed for many applications. Kumar et al. [18] proposed the Fuzzy with Cohort Intelligence (F-CI) model to categorize the nature of breast cancer type. Also, the F-CI approach utilized the breast cancer diagnostic dataset for detecting the disease. Moreover, the accuracy of the classification measure is improved by the meta-heuristics model but, it is very complicated to complete the entire process.

The identification and categorization of breast tumors in the premature phase is helpful to the patients for taking proper treatment. In this, Khan et al. [16] established the pre-trained Convolutional Neural Network (CNN) model in the Visual Geometry Group Network (VGGNet) to identify the cancer tissues in the breast. Here, the efficacy of the developed $\mathrm{CNN}$ in the VGGNet model is analyzed with standard benchmark datasets. However, this model is processed with high operating time and achieves low efficiency.

Persistent ductal carcinoma tumor attacks the breast muscles by extinguishing the milk canal; it is the most frequent kind of breast tumor in women. Around $80 \%$ of patients suffering from breast tumors and approximately $66.6 \%$ of patients are above 55 years. Toğaçar et al. [39] developed CNN with an autoencoder model that helps to process the linear analysis and detect the breast tumor from MRI images in an earlier stage. But, it has attained lower performance for predicting the tumors.

To improve the disease detection exactness score, Patil and Biradar [27] have been developed a firefly updated chicken-based Chicken Swarm Optimization with Convolutional Recurrent Neural Network (FC-CSO-CRNN) mechanics. This hybrid model is efficient in predicting diseases in complex MRI images. Finally, it has reported better accuracy while comparing conventional RNN and CNN models; however, the designing process has taken more time than the conventional RNN and $\mathrm{CNN}$ approach.

In another case, an Optimized Artificial Neural Network (OANN) was designed by Supriya and Deepa [38] for disease detection. The key merit of this proposed model is it has required less energy to run the process. However, attaining the best result has required less energy to run the process.

The technique Multi-Verse Optimizer (MVO) and Gradient Boosting Decision Tree (GBDT) was designed by Junior et al. [13] to detect breast cancer into dual groups that are benign and malignant. Finally, the key parameters are calculated and compared with previous work and recorded low error. But it has consumed more energy.

Zheng et al. [48] have presented a DL aided Effectual Adaboost Algorithm (DLA-EABA) to detect breast cancer in an earlier stage to minimize the death rate. Also, the classification of tumor was also done by convolution neural model simultaneously flaw estimation paradigm was also implemented to minimize the flaw and enhance the accuracy. Hence, it has gained 96.5\% specificity; however, it is expensive in cost while comparing other approaches.

Zhang et al. [47] have designed the CNN model to detect breast cancer. Here, the datasets are validated under a tenfold-based cross-validation approach. Moreover, this approach has taken a few seconds to run the execution, however, the main demerit in this model is low accuracy of detection.

In another research, Deep Neural Network with Support assessment (DNNS) mechanism has been implemented by Vaka et al. [40] to predict breast cancer in the early stage. To check the proposed design, diverse kinds of experimental validation or performed. Finally, it has gained the finest accuracy while comparing previous schemes. However, it has taken more duration to execute the process.

Melekoodappattu and Subbian [23] have projected a novel Extreme Learning Machines with Fruit fly Optimization Algorithm (ELM-FOA) [23] to predict breast cancer; moreover, to values the presented model, the mammogram datasets are taken into account. Subsequently, it is noted that the projected schema has gained $100 \%$ precision and $97.5 \%$ sensitivity. But is complexity in design. 
Piantadosi et al. [29] designed deep CNN (DNN) for automatic affected region segmentation in breast images. In addition, the $\mathrm{CNN}$ is structured in $3 \mathrm{D}$ form to get the exact result, so based on the disease severity graphs are attained. Hence, it has helped to get accurate results in all complicated databases. However, it has required more time to complete the process.

In another case, to remove the training errors and enhance the affected region's prediction, Ghasemzadeh et al. [9] have developed Gabor-wavelet transform (GWT) to filter the mammogram breast images. Hereafter, the feature selection is made by a cross-validation model; still, it has attained average prediction accuracy as $93 \%$. Also, if the data is complex, it has reported a high error rate.

Yavuz and Eyupoglu [45] have designed a Generalized Regression Neural Network (GRNN) to build a proficient detection system to identify the disease features from the dataset. It has gained $97 \%$ of disease diagnosis accuracy, which quite better than other previous models. Also, the disease is predicted by analyzing the blood characteristics. However, it has required wide resources for designing the prediction system.

Shen et al. [36] has proposed end-to-end training DL paradigm as a cancer prediction system in the breast cancer dataset. This projected algorithm can train the large dataset and gain better accuracy for disease classification. However, if the data is too large, it requires more time.

Yala et al. [44] developed the Deep Learning (DL) model based on mammography images, which is utilized to analyze breast cancer risk factors. This method utilized the mammograms for many patients are employed for processing like training, evaluation, and testing. In addition, MRI breast images also validated in this model, it has gained wide accuracy. However, it has reported a high error rate.

Breast cancer risk prognosis approaches depend on density calculations and questionnaires. Here, Dembrower et al. [5] established the risk score model based on DL for analyzing breast cancer risk factors. Moreover, the developed approach calculated the disease's dense area, risk score, and density. But it has consumed more energy.

\section{System model and problem statement}

The image processing methods are utilized in medical research for several applications like computer-aided detection, mammography, breast MRI, and breast ultrasound. Many kinds of research are done for the primary revealing of breast tumors to begin the treatment and enhance the prospect of endurance; most of the studies focus on mammogram imageries. But, in some cases, the mammogram images contain a threat of the wrong prediction that may cause danger to the patient's fitness.

The system model for detecting breast cancer and the issues are mentioned in Fig. 1. Thus, there is a requirement to find substitute models that are inexpensive, harmless, simpler to execute and operate with dissimilar data sets that can generate a trustworthy prediction. To overcome prediction issues, this research introduced an innovative deep learning technique. Here, the introduced model utilized MRI breast image datasets that have high performance for classifying breast cancer.

\section{Proposed BPBRW with HKH-ABO mechanism}

In general, breast cancer affects women's breast cells and causes tumor formation, which reduces the health of women. According to the 2018 report, $50 \%$ of Indian women are affected 


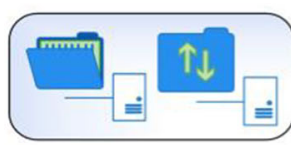

Image acquisition

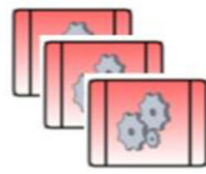

Processing
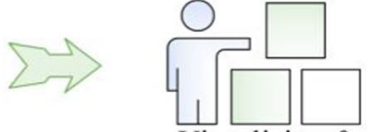

Visualizing \& reporting

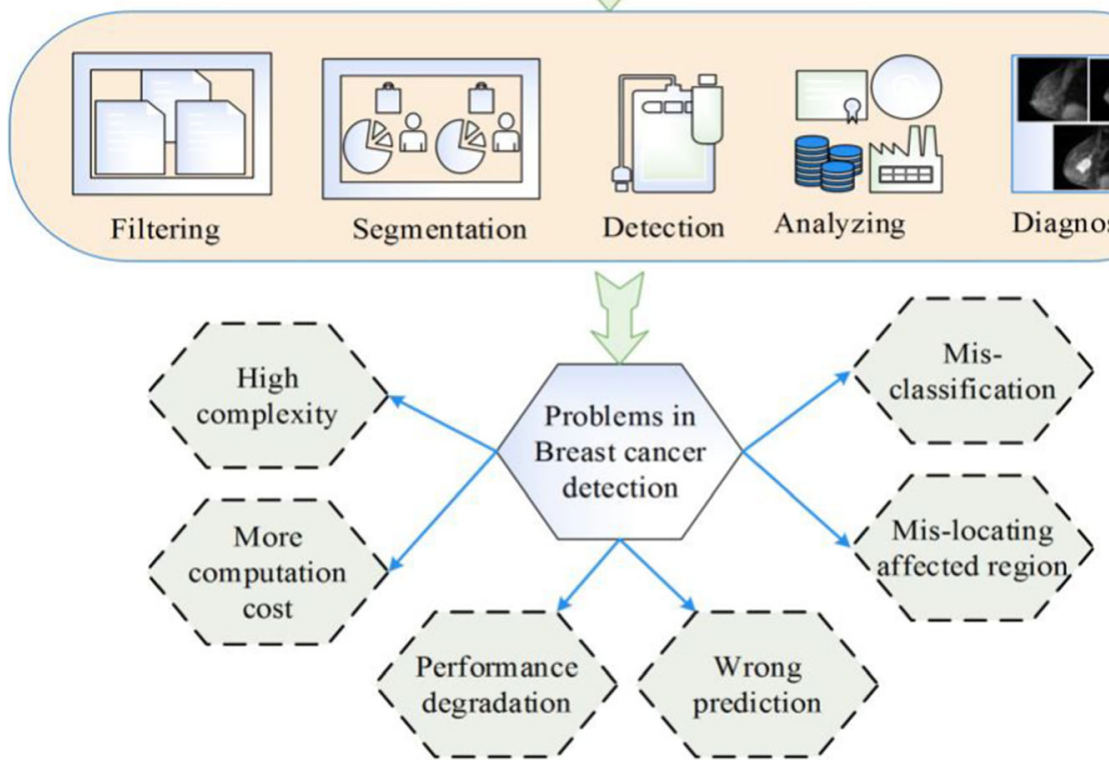

Fig. 1 System model and problems associated with breast cancer detection

by breast cancer and $58 \%$ of deaths have occurred worldwide. So, this research developed the BPBRW with HKH-ABO approach for predicting breast cancer in an earlier stage. Initially, breast MRI images are utilized in the system for training and testing, which are undergoing the preprocessing process using a novel Wienmed filter to eliminate the undesired noise components in the MRI. Consequently, the developed BPBRW with HKH-ABO model is processed over the breast MRI dataset for categorizing the cancer cells and their types in an earlier stage.

In addition, the classification accuracy is enhanced using the developed BPBRW with HKHABO mechanism. Hence, the developed approach predicts and classifies the breast tumor in an initial stage. The processing steps of the developed strategy are demonstrated in Fig. 2.

\subsection{Image acquisition}

The proposed BPBRW with HKH-ABO has utilized breast MRI images for classifying the disease types. Here, 1000 breast MRI images [11] are collected from the net source, processed on the proposed BPBRW with HKH-ABO approach for categorizing the cancer types as benign or malignant. The collected images involve 735 malign images and 265 benign images with $256 \times 256$ resolution and less than $2 \mathrm{~mm}$ thickness. Thus, the collected images are utilized as the dataset that is processed in the developed manner in the form of training and 


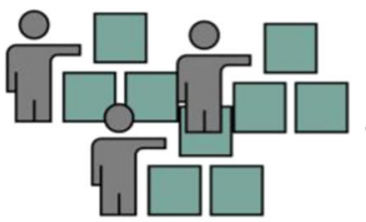

Breast MRI dataset

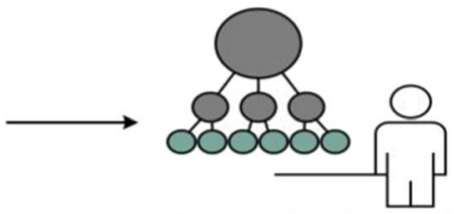

Proposed BPBRW with

$\mathrm{HKH}-\mathrm{ABO}$

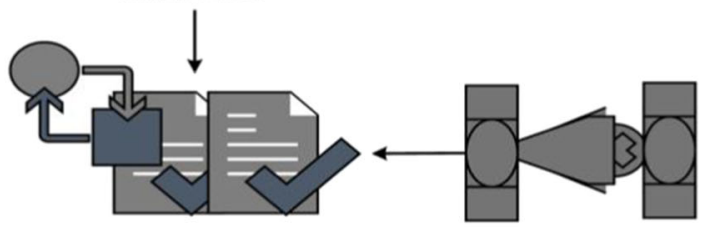

Preprocessing

Wienmed filter

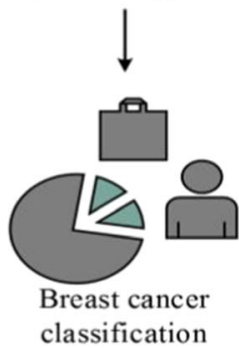

classification

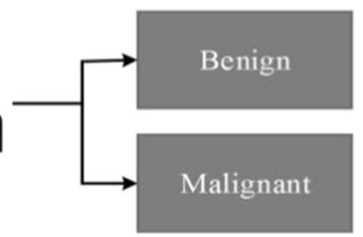

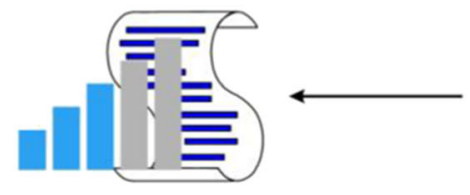

Performance evaluation

Fig. 2 Proposed BPBRW with HKH-ABO model

testing. The collected MRI encloses undesirable errors or noises, which are removed by the preprocessing process. The dataset specification is shown in Table 1.

\subsection{Pre-processing process}

The collected MRI breast images contain unpredictable and irrelevant noises or errors that minimize the image diagnosis and affect the image contrast resolution. Thus, the preprocessing process aims to upgrade the performance of the following stages using some conversions to set up the MRI and make them appropriate for the succeeding processing stages. In this work, a novel Wienmed filter is utilized for removing the inappropriate noise and undesirable portions in the image background, thereby confining the high or low frequencies that improve or perceive the image boundaries. Here, the Wienmed filter is the collective form of two filters that are Wiener [7] and median filters [35]. These two filters are combined to reduce noise distribution and errors in MRI breast images effectively. The most important intent of such a filter is to replace the noisy and neighborhood image pixels, and formerly they were arranged depending on the image's intensity. This preprocessing phase improves the MRI breast image portion that comprises undesired distortions or improves numerous image attributes that are noteworthy for additional processing. The MRI dataset is processed on the Wienmed filter, and finally, the preprocessed MRI image is achieved. In this, the mean value $m$ of each pixel has $(P Q)$ sized mask matrix is computed using Eq. (1),

$$
m=\frac{1}{P Q} \sum_{p, q \in A} a(p, q)
$$


Table 1 Dataset specification

\begin{tabular}{|c|c|}
\hline Number of samples & 1000 \\
\hline Benign cases $^{\mathrm{a}}$ & 265 \\
\hline Malign cases ${ }^{b}$ & 735 \\
\hline Image resolution & $256 \times 256$ \\
\hline Modality & MR \\
\hline Slice thickness & $<2.0 \mathrm{~mm}$ \\
\hline
\end{tabular}

${ }^{a}$ normal cells

b abnormal cells

Where, $p, q$ is represents the pixel dimensions of each image, $a$ denotes each image and $A$ is an image. Also, the Gaussian noise variance is given in Eq. (2), $\sigma^{2}$ is the variance of Gaussian noise in the image and $n$ is the noise features. Here, the present noise features were traced.

$$
\sigma^{2}=\frac{1}{P Q} \sum_{p, q \in A} n(p, q)-m^{2}
$$

The Wiener equation for the new image pixel is given in Eq. (3), here $w$ is the wiener variable. Here the traced noise features were removed from the dataset.

$$
w(p, q)=\sigma^{2}[n-a(p, q)]
$$

Thus, the developed Wienmed filter effectively removes the undesired noises in the MRI breast images and the attained images are moved to the classification process.

\subsection{Breast cancer classification}

The filtered MRI dataset is processed using Back Propagation Boosting Recurrent Wienmed (BPBRW) with HKH-ABO model that can classify the disease types to identify the severity of the breast cancer. Here, the HKH-ABO model enhances the classification accuracy, which is initiated in the classification layer of BPBRW. Also, HKH-ABO is the combined model of Krill Herd Optimization (KHO) [25] and African Buffalo Optimization (ABO) [14]. Moreover, the goal of hybridization is to improve the efficiency of disease classification. Initially, the BPBRW model is developed for classifying the breast tumor using the dataset that is a neural network system based on decision trees. It is a group learning strategy utilized for categorization, regression, and performing other functions, which is worked based on the creation of decision trees on training time and producing the classification of the distinct trees. Initially, the filtered breast MRI dataset is categorized as training and testing samples. Here, the testing samples are utilized for processing that is denoted in Eq. (4),

$$
D_{s}=\left\{\left(P_{m}, Q_{m}\right)\right\}, m=1,2, \ldots, N
$$

Where, the breast MRI for $m$ numbers of patients is mentioned as $P_{m}=$ $\left(p_{m}^{1}, \ldots \ldots \ldots \ldots . . . p_{m}^{M}\right)$ with $M$ features and the breast cancer tumor is mentioned as $Q_{m}$ that includes the details about tumor like benign and malignant.

Moreover, BPBRW is an arithmetical procedure to cluster the data points in functional sets. For a larger dataset with more variables, it is difficult to cluster the data since all the variables cannot be considered Hence, the procedure could also provide a specific chance that the data point fit in a specific group. In this network, the Long-Short Term Memory (LSTM) [8] is the 
memory unit that eliminates or includes the data to the cell state that is sensibly controlled by structures named gates. As the infection increases, the degree of malignancy will differ after some time. The present tumor size depends on the earlier data of the tumor. Consequently, if joining and developing the history at past time steps, the prediction of tumor size would be better. Also, the LSTM arrangement offers a gating tool for gathering data to evade long-term reliance issues that comprise an input, hidden, and output gate. Thus, the overall process of the developed method is explained in Fig. 3.

Let $i_{t}$ be the input sequence with sigmoidal activation function, $h_{t-1}$ is the output of last time step, and $x$ is the input data at present step that is given in Eq. (5).

$$
a_{t}=b+y h_{t-1}+x i_{t}
$$

In this, the filtered input images are given to the BPBRW model that is analyzed the diseases. Also, the second part is the output $h_{t}$, image feature is denoted as $y$, predicting the disease features from the trained data is mentioned in Eq. (6),

$$
h_{t}=Q_{m}\left(P_{m}\right)
$$

Moreover, the disease part is identified in this function and the developed HKH-ABO model is initiated in this layer that is given to the output layer. Initially, the fitness of the krill herd $\left(K_{p n}^{*}\right)$ is utilized for enhancing the disease classification that is expressed using Eq. (8).

$$
K_{p n}^{*}=\frac{K_{f}^{*}-K_{g}^{*}}{K_{w}^{*}-K_{b}^{*}}
$$

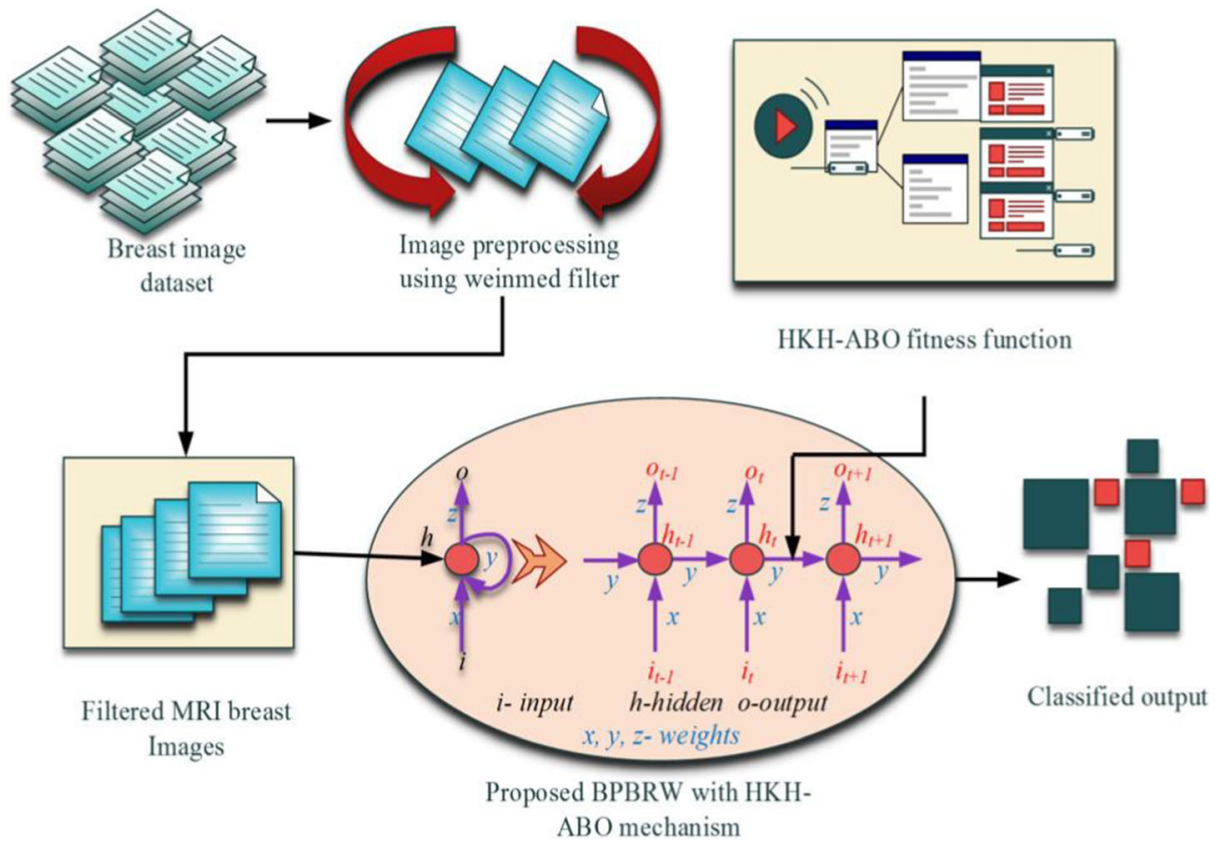

Fig. 3 Working process of the developed model 
Here, $K_{w}^{*}, K_{b}^{*}$ denotes the normal and abnormal feature of each MRI data, $K_{f}^{*}$ is the predicted normal features and $K_{g}^{*}$ is the predicted disease features. Let $C_{b}$ is the classification parameter of the $p^{\text {th }}$ individual patients. The coefficient is well-defined as a target $I$ leads the resolution to the global optimum and it is more efficient than other individuals. The value of $C_{b}$ is demarcated in Eq. (8),

$$
C_{b}=2\left(\operatorname{ran}+\frac{I}{I_{\max }}\right)
$$

Where, ran is a random value lies among 0 and 1 that is improving the search, $I$ is the real repetition numeral and $I_{\max }$ is the greatest amount of repetitions. The prediction of the disease for individual $p^{\text {th }}$ patient is given in Eq. (9),

$$
w_{f+1}=\frac{w_{f}+m_{f+1}}{\lambda^{*}}
$$

Where, $w_{f}$ represent the specific image in processing and $m_{f}+1$ denotes the features of the particular image, and $\lambda^{*}$ trained disease features. Consequently, the attained images are given to the output layer and that provided the classified output. Thus, the output sequence $o_{t}$ (classified images) is computed using Eq. (10),

$$
o_{t}=\left(c^{*}+z h_{t}\right) w_{f+1}
$$

Where, $c^{*}$ denotes the benign images, $h$ denotes the weight of images, iteration count is denoted as $t$ and $z$ denotes the malignant images. Hence, the proposed BPBRW with the HKHABO model has classified breast cancer using an MRI dataset. Moreover, the classified MRI images are performed on the feature extraction process for reducing dimensional errors.

\subsubsection{Feature extraction}

The feature extraction stage provides the image information essential for carrying out the operation based on certain applications. The feature extraction by Grey level Co-occurrence Matrix (GLCM) [12] is deliberated as substantial for the classification process. Here, GLCM is set up by utilizing the image grayscale values and reveals the brightness of the pixels in an image. In this work, second-order image texture features are expressed for extracting the statistical texture features specifically energy, correlation, entropy, contrast, homogeneity, etc., are calculated. The extracted features should be selected sensibly to perform the desired classification task in that selected region instead of considering the whole region. Here, the features are extracted to categorize the kind of breast tissue and to differentiate either normal or cancerous breast by GLCM. Hence, the features are extracted in an MRI image that can enhance the resolution of the images. Moreover, the classified images are given to the HKHABO model to enhance the images' classification accuracy. Hence, the interior process of the proposed methodology is explained in algorithm 1.

The complete process of the BPBRW with HKH-ABO workflow is detailed in Fig. 4. Finally, the proposed BPBRW with $\mathrm{HKH}-\mathrm{ABO}$ approach effectively classifies the disease types as benign and malignant. Also, it enhanced the classification accuracy than other models. The training ratio is $500 \mathrm{MRI}$ images and the testing ratio is 25 samples. During the process, if dataset imbalance issues are raised, then it has been solved by tuning the HKH-ABO parameter to the other levels. 


\section{Algorithm 1: BPBRW with HKH-ABO mechanism}

Input: MRI breast images

Output: Classified the breast images

Start

\{ int $D(p, q)$ //breast MRI dataset

dataset training

Training $\rightarrow p_{m}$

// input images dataset

for all $p_{m}$

Pre-processing

Filtering using eqn. (3)

// here, the training errors are removed

classification

$\forall B_{p q} \rightarrow f(p, q) \rightarrow$ input layer

//HKH-ABO is initiated in the hidden layer

Calculate the fitness function

Calculate $p_{m}=w_{f+1}$

//initiating the disease prediction model

Locate the starting point of cancerous region $b_{j}$ using eqn. (6)

Trace for the identical region using eqn. (7)

Prediction of cancerous region

Classifying the cancer types

If $\left(C_{b}<<0\right)$ then benign

else

If $\left(C_{b}<<1\right)$ then malignant

end if

Extract second order statistical features for classification

end for

Enhanced classification accuracy

Output best solution

\}

Stop 


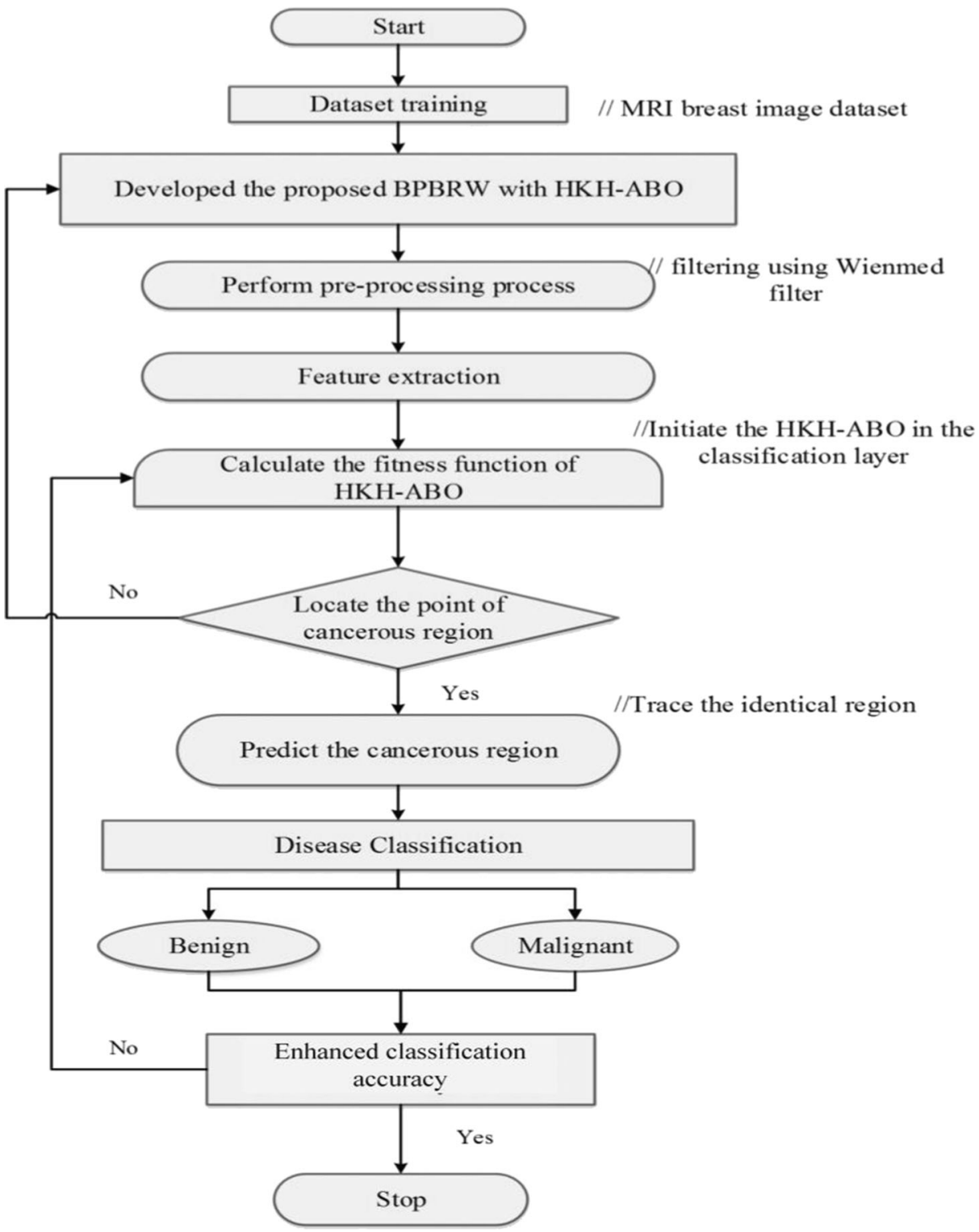

Fig. 4 Block diagram of the BPBRW with HKH-ABO model

\section{Results and discussion}

The proposed BPBRW with HKH-ABO approach is executed using Python, and the performance parameters are calculated. The proposed work intends to predict the breast cancer prototype by utilizing an optimized deep learning mechanism. Here, image preprocessing, affected part division, feature abstraction, and classification are the useful parts of the recommended breast cancer recognition system. The MRI breast image dataset is collected, and the acquired images undergo preprocessing using a novel Wienmed filter that eliminates 
the noise in the MRI image. The BPBRW with HKH-ABO mechanism is utilized to categorize the cancer types as either Benign or malignant and enhance the classification accuracy with a minimum false discovery rate. The performance is validated with existing accuracy, Recall, precision, F1-score, sensitivity, specificity, and Error Rate. The experimental results have shown improved outcomes than the conventional model. The key reason for that improvement is the proposed is designed with dual hybridization, that is, deep learning and optimization. Hence, that helps to attain the best result. Also, the model has any drawbacks, then those issues are cleared by the other algorithm during hybridization.

\subsection{Case study}

Consider a trained MRI breast image sample to illustrate the experimentations to interpret the results. Here, the collected MRI breast dataset is initially taken for the training and testing process by the proposed technique. Also, the input images undergo a preprocessing operation to eliminate the MRI distortion or noise that is done using a Wienmed filter. The filtered images are given to the proposed BPBRW with the HKH-ABO model that can classify the breast cancer types. Here, the HKH-ABO mechanism operates over the BPBRW model to find the area of the cancerous spot. The second-order features are then extracted to classify the type of cancer. Hence, the benign and malignant type of breast cancer is classified using the developed BPBRW with the HKH-ABO approach. The classified output images are shown in Fig. 5.

Additionally, the cancer cell images for the classified images are identified in Fig. 6. The benign and malignant forms of the cells are represented, which can help identify the severe breast cancer case. If the detected region of the breast is malignant, it is determined to be a severe case. Hence, it shows that immediate treatment is required for the patient to get cured of this disease like surgery and chemotherapy.

This research developed BPBRW with HKH-ABO model processed on the MRI breast dataset for classifying the disease types. The utilization of hybrid optimization has increased the efficiency of the disease classification, which is categorized the images as benign or malignant.

There is the vulnerability of adversarial attacks in all digital applications because of wireless and cloud environments. So, if the disease prediction system was attacked, it interrupted the entire system function. So that defense mechanism is needed to keep the system away from malicious events. Hence, this kind of adversarial attack was defended by implementing the security algorithm or locking framework [32].

In the future, to make this proposed algorithm an effective model of breast cancer diagnosis, this security mechanism will be planned to implement.

\subsection{Performance measures}

Some of the metrics used to validate the projected model's efficacy are accuracy, Recall, precision, F1-score, sensitivity, and specificity. The effectiveness of the projected work is validated with existing methods like Firefly updated chicken-based Chicken Swarm Optimization with Convolutional RNN (FC-CSO-CRNN) [27], Optimized Artificial Neural Network (OANN) [38], and MVO-GBDT [13].

The basic factors to be computed as true positive $\left(t_{p}\right)$, false positive $\left(f_{p}\right)$, false negative $\left(f_{n}\right)$, and true negative $\left(t_{n}\right)$ standards. In this, $\left(t_{p}\right)$ indicates the measure of belongings in which the 


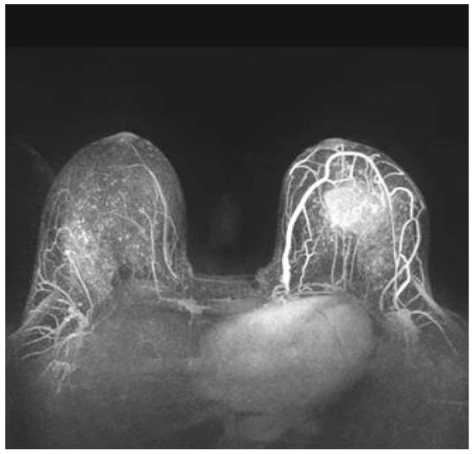

(a) Malignant

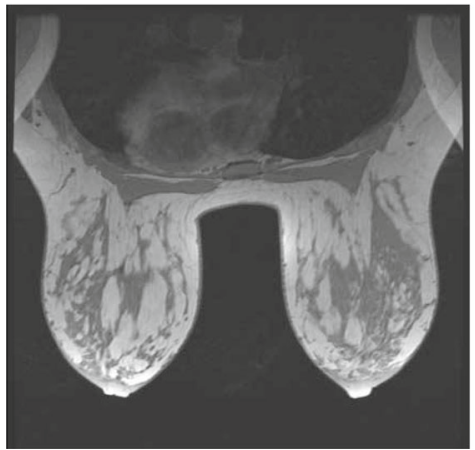

(c) Benign

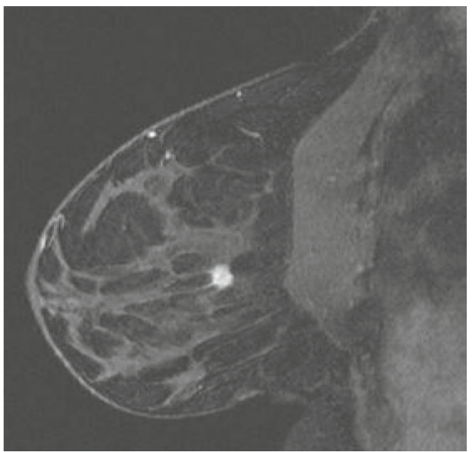

(b) Malignant

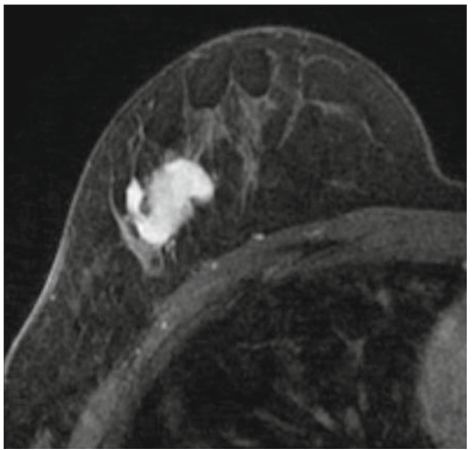

(d) Malignant

Fig. 5 Classified MRI breast images

proposed method identifies the cancer region appropriately, $\left(t_{n}\right)$ specifies the circumstances, here a normal region is classified appropriately, $\left(f_{p}\right)$ represents the normal regions that are wrongly considered as the cancerous region, and the state in which the cancer region noticed as a normal is signified as $\left(f_{n}\right)$.

\subsubsection{Accuracy calculation}

It is the degree of correctness of the system model's functioning. In general, it is the fraction of properly expected observance to the entire observations. The accuracy is expressed using Eq. (15),

$$
\text { Accuracy }=\frac{t_{p}+t_{n}}{t_{p}+t_{n}+f_{p}+f_{n}}
$$

In this, the measurement of the accuracy for BPBRW with the HKH-ABO approach is evaluated with prevailing approaches like OANN, FC-CSO-CRNN, and MVO-GBDT. The graphical representation of accuracy comparison is shown in Fig. 8.

The accuracy measure comparison of the proposed BPBRW with the HKH-ABO technique and various techniques like FC-CSO-CRNN, OANN, and MVO-GBDT are discussed in Table 2. The result shows that the projected scheme achieves higher 


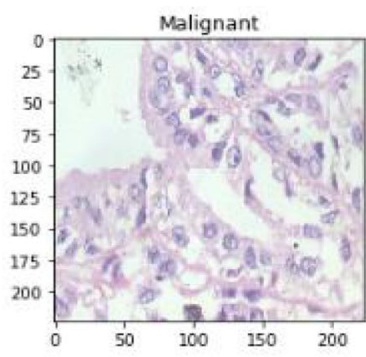

(a)

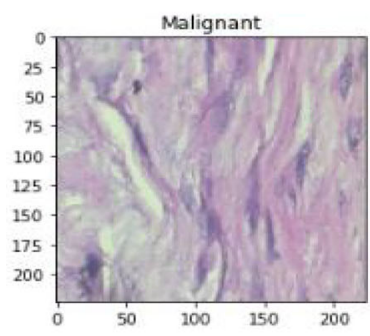

(d)

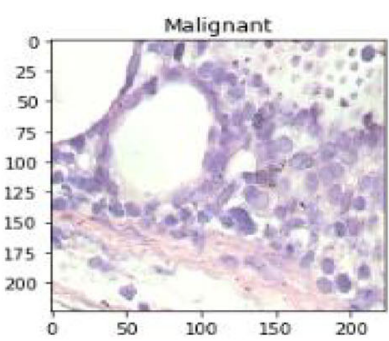

(b)

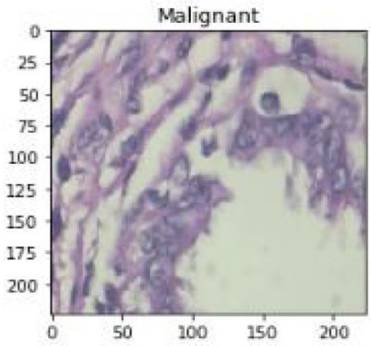

(e)

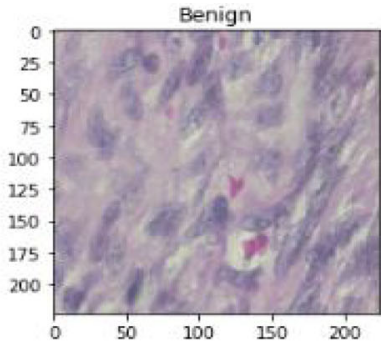

(c)

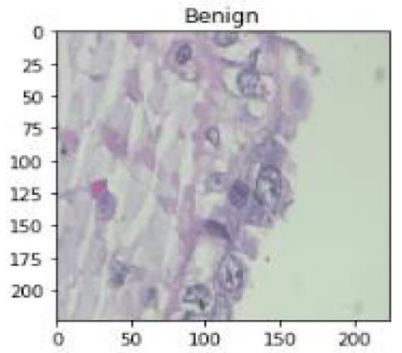

(f)

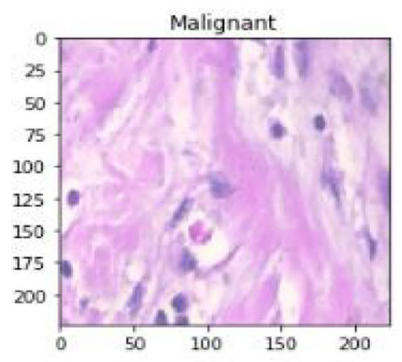

(g)

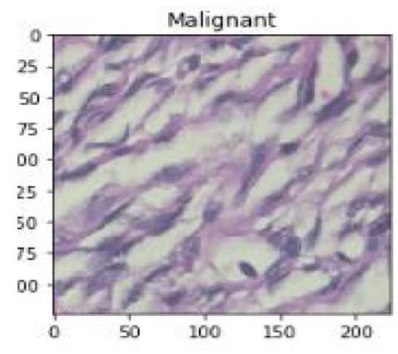

(h)

Fig. 6 Classified segments of the breast cancer cell images

Table 2 Assessment of accuracy

No. of samples Accuracy A $^{\mathrm{a}}$ )

\begin{tabular}{llll}
\hline $\begin{array}{l}\text { FC-CSO-CRNN } \\
\text { [27] }\end{array}$ & OANN [38] & MVO-GBDT [13] & BPBRW with HKH-ABO [Proposed] \\
\hline 93.59 & 98 & 97.52 & 99.6 \\
93.28 & 97.68 & 97.28 & 99.08 \\
92.87 & 97.45 & 96.97 & 99 \\
92.56 & 96.90 & 96.65 & 98.97 \\
92.27 & 96.54 & 96.18 & 98.56 \\
\hline
\end{tabular}

a identify the effectiveness of the proposed approach 
Table 3 Comparison of precision

\begin{tabular}{lllll}
\hline \multirow{2}{*}{ No. of samples } & \multicolumn{3}{l}{ Precision $^{\mathrm{a}}(\%)$} & \\
\cline { 2 - 5 } & FC-CSO-CRNN & OANN & MVO-GBDT & BPBRW with HKH-ABO [Proposed] \\
\hline 5 & 97.01 & 98.03 & 98 & 99.9 \\
10 & 96.75 & 97.98 & 97.78 & 99.46 \\
15 & 96.50 & 97.05 & 97.46 & 99.05 \\
20 & 96.04 & 96.46 & 96.56 & 98.89 \\
25 & 95.97 & 96.29 & 96.07 & 98.65 \\
\hline
\end{tabular}

a Identify the accurate prediction of diseases

accuracy of $99.6 \%$ than the existing models. Here 500 MRI images are taken as training samples and 25 images are used for testing. Hence, the parameters are calculated for 25 samples.

\subsubsection{Precision}

Precision is evaluated as the number of correct positive estimates alienated by the overall positive estimates. It is the proportion of precise diagnosis of the affected region to be cancer that is computed using Eq. (16),

$$
P=\frac{t_{p}}{t_{p}+f_{p}}
$$

The precision comparison of the proposed BPBRW with HKH-ABO strategy and various techniques like FC-CSO-CRNN, OANN, and MVO-GBDT are discussed in Table 3. Here, the existing methods attained nearly 97 and $98 \%$ precision values only. Thus, the proposed BPBRW with HKH-ABO has attained $99.9 \%$ high precision value than other models that are shown in Fig. 9. The confusion matrix of the developed technique is represented in Fig. 7.

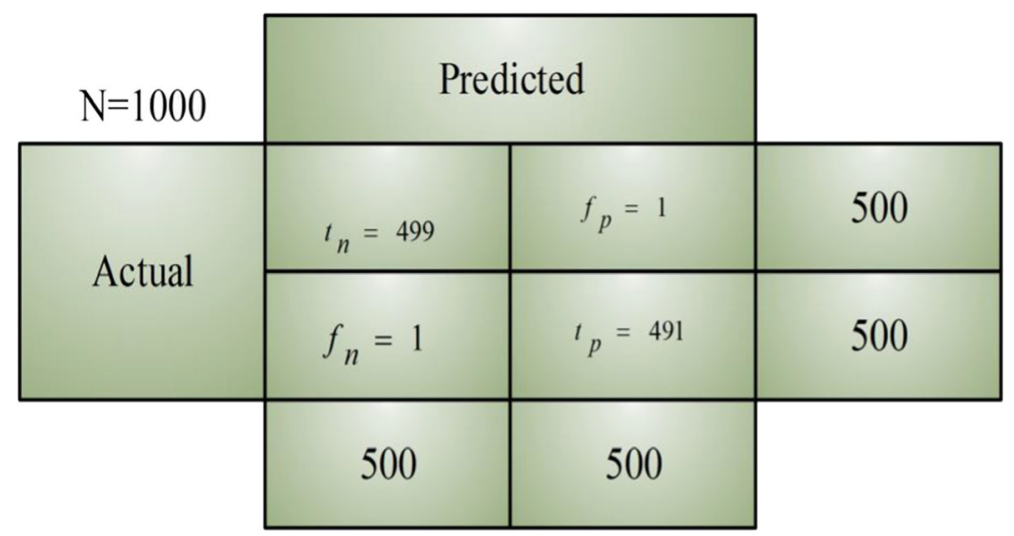

Fig. 7 Confusion matrix 


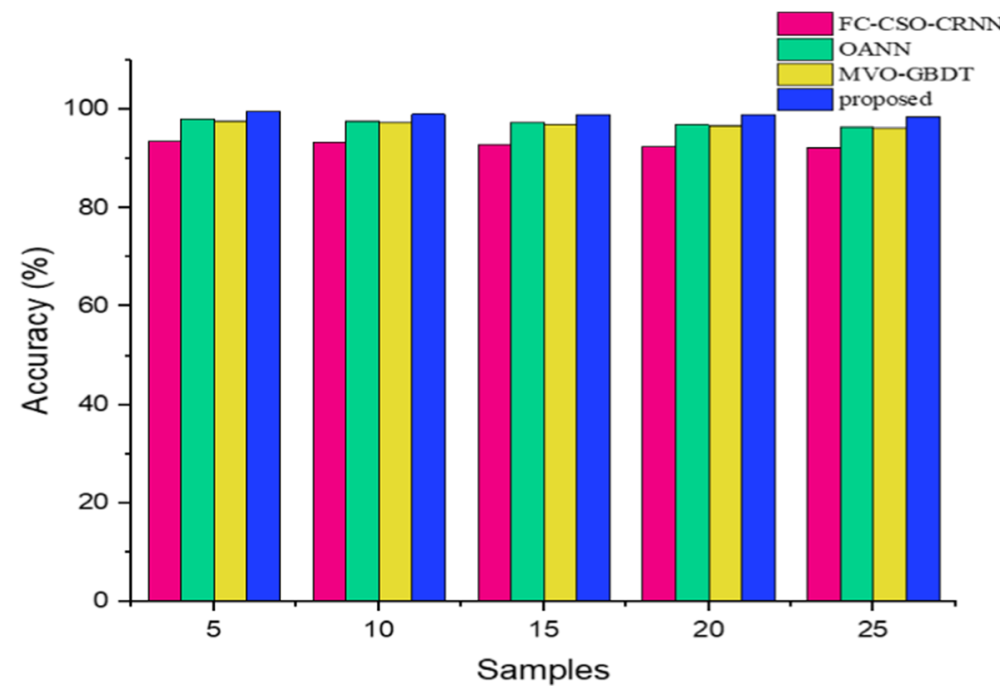

Fig. 8 Accuracy assessment

\subsubsection{Recall}

The Recall is intended as the amount of correct positive forecast to the overall true positives and false negatives. It expresses what proportion of predictions has been correctly diagnosed as cancer that is represented using Eq. (17).

$$
R=\frac{t_{p}}{t_{p}+f_{n}}
$$

The comparisons of Recall for the proposed BPBRW with HKH-ABO strategy with various other techniques like FC-CSO-CRNN, OANN, and MVO-GBDT, which are discussed in Table 4.

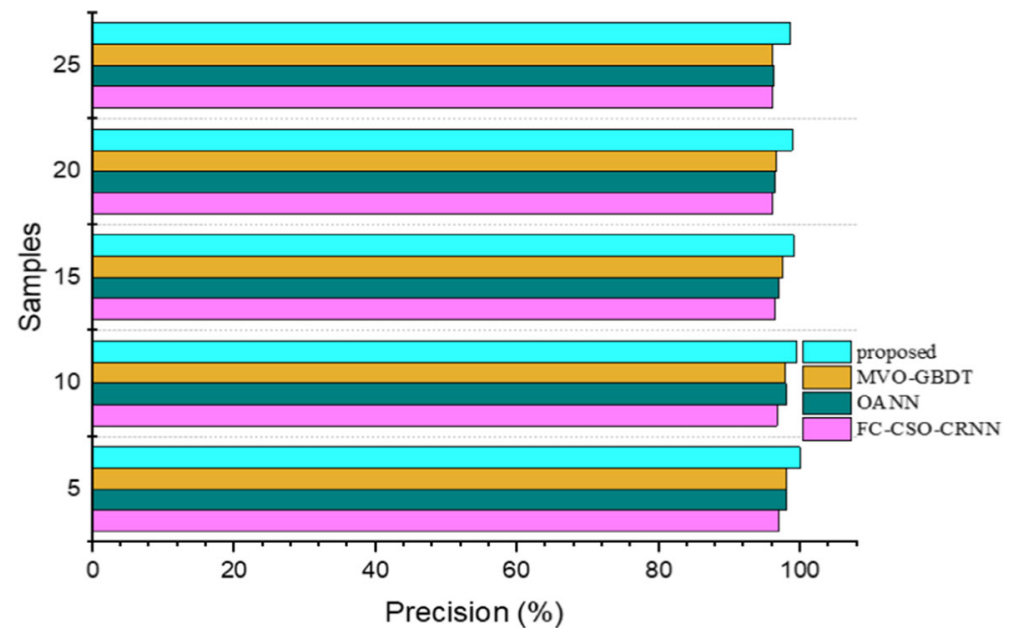

Fig. 9 Comparison of precision 
Table 4 Comparison of recall

\begin{tabular}{lllll}
\hline No. of samples & \multicolumn{3}{l}{ Recalla $\left.^{\mathrm{a}} \%\right)$} \\
\cline { 2 - 5 } & FC-CSO-CRNN & OANN & MVO-GBDT & BPBRW with HKH-ABO [Proposed] \\
\hline 5 & 96.89 & 97.59 & 98 & 99.9 \\
10 & 96.56 & 97.64 & 98.06 & 98.67 \\
15 & 96.09 & 97.53 & 97.97 & 98.04 \\
20 & 95.86 & 96.83 & 96.69 & 97.09 \\
25 & 95.35 & 96.94 & 96.84 & 97.57 \\
\hline
\end{tabular}

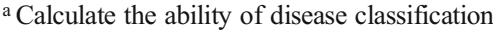

\subsubsection{Sensitivity}

It is a degree that measures the number of true positives, which are predicted precisely. It is the probability of identifying the benign type of cancer that is measured using Eq. (18) as,

$$
\operatorname{Sen}=\frac{t_{p}}{t_{p}+t_{n}}
$$

The proposed approach calculated the sensitivity value based on the number of training samples that are evaluated using prevailing approaches.

The sensitivity measure of the proposed BPBRW with HKH-ABO strategy is compared with various other techniques like FC-CSO-CRNN, OANN, and MVO-GBDT. The existing approaches attained a lower sensitivity value that is discussed in Table 5.

The result shows that the projected scheme achieves high sensitivity of $97.7 \%$ than the existing ones and the sensitivity comparison is specified in Fig. 11.

\subsubsection{Specificity}

It is the degree that measures the number of true negatives, which are identified exactly. Also, it is utilized to identify the malignant type of cancer computed using Eq. (19) as,

$$
\text { Spec }=\frac{t_{n}}{f_{p}+t_{n}}
$$

Table 5 Comparison of sensitivity

\begin{tabular}{lllll}
\hline \multirow{2}{*}{ No. of samples } & \multicolumn{2}{l}{ Sensitivity $^{\mathrm{a}}(\%)$} & \\
\cline { 2 - 5 } & FC-CSO-CRNN & OANN & MVO-GBDT & BPBRW with HKH-ABO [Proposed] \\
\hline 5 & 97.85 & 96.89 & 96.45 & 98.68 \\
10 & 97.96 & 96.58 & 96 & 98.43 \\
15 & 97.43 & 96.05 & 95.36 & 98.07 \\
20 & 97.37 & 96.23 & 95.08 & 97.95 \\
25 & 97.01 & 96.03 & 95.01 & 97.7 \\
\hline
\end{tabular}

a Ability to identify those with diseases 
Table 6 Comparison of Specificity

\begin{tabular}{lllll}
\hline \multirow{2}{*}{ No. of samples } & \multicolumn{2}{l}{ Specificity $(\%)$} & \\
\cline { 2 - 5 } & FC-CSO-CRNN & OANN & MVO-GBDT & BPBRW with HKH-ABO [Proposed] \\
\hline 5 & 93.67 & 86.49 & 93.85 & 95.78 \\
10 & 92.89 & 87.56 & 93.76 & 94.57 \\
15 & 92.56 & 88 & 92.96 & 93.92 \\
20 & 92.39 & 88.87 & 93.02 & 93.7 \\
25 & 92.21 & 88.21 & 92 & 92.5 \\
\hline
\end{tabular}

a Ability to identify those without diseases

The specificity measure for the proposed strategy is compared with various existing techniques like FC-CSO-CRNN, OANN and MVO-GBDT models. Here, the obtained outcomes are discussed in Table 6.

The result shows that the projected scheme achieves high specificity of $92.5 \%$ than the existing ones and the specificity comparison is illustrated in Fig. 12.

\subsubsection{Error rate}

The error rate is calculated for identifying the variation among the exact prediction and actual prediction of diseases by the proposed approach, which is calculated using Eq. (20),

$$
E_{r}=\frac{\left(f_{p}+f_{n}\right)}{\text { total }}
$$

The error rate of the developed BPBRW with the HKH-ABO approach is validated with existing approaches that are given in Table 7. Here, the prevailing methods like FC-CSO$\mathrm{CNN}$, OANN, and MVO-GBDT approaches are attained a high error rate as $0.25 \%, 0.8 \%$, $1.7 \%$, respectively.

The proposed BPBRW with HKH-ABO approach has attained a lower error rate of $0.12 \%$ for predicting breast cancer. The validated results for error rate are represented in Fig. 13. The separate form of $\mathrm{KHO}$ and $\mathrm{ABO}$ models has low efficiency for classifying breast cancer. The hybrid forms of these methods attain high efficiency, as mentioned in Table 8. Moreover, theses optimizations are implemented in same environment to attain effectiveness exactly. To show the difference between hybrid optimization and other optimization, Table 9 is tabulated, which has attained the best outcomes for the proposed hybrid method. Also, the DL approaches with other optimization model is shown in Figs. 8, 9, 10, 11, 12, 13. In all cases,

Table 7 Comparison of error rate

\begin{tabular}{lllll}
\hline No. of samples & \multicolumn{2}{l}{ Error rate $(\%)$} & \\
\cline { 2 - 5 } & FC-CSO-CRNN & OANN & MVO-GBDT & BPBRW with HKH-ABO [Proposed] \\
\hline 5 & 0.25 & 0.8 & 1.7 & 0.12 \\
10 & 0.32 & 1.3 & 2.9 & 0.15 \\
15 & 0.5 & 1.8 & 3.8 & 0.17 \\
20 & 0.77 & 2.5 & 4.2 & 0.2 \\
25 & 1.5 & 3 & 5.7 & 0.26 \\
\hline
\end{tabular}


Table 8 Comparison of optimization in a separate manner and hybrid manner

\begin{tabular}{|c|c|c|c|c|c|}
\hline \multirow[t]{2}{*}{ Parameters } & \multirow[b]{2}{*}{$\mathrm{KHO}^{\mathrm{a}}$} & \multirow[b]{2}{*}{ FC-CSO [27] } & \multirow[b]{2}{*}{ MVO [13] } & \multicolumn{2}{|c|}{ Optimization methods } \\
\hline & & & & $\mathrm{ABO}$ & Proposed [HKH-ABO] \\
\hline Accuracy $(\%)$ & 79.5 & 80 & 85.6 & 85.67 & 99.6 \\
\hline Precision (\%) & 82 & 81.2 & 84 & 87 & 100 \\
\hline Recall (\%) & 80.08 & 82 & 83.5 & 86.46 & 99.9 \\
\hline Specificity (\%) & 85.6 & 67 & 81 & 82.45 & 95.78 \\
\hline Sensitivity (\%) & 84.56 & 85 & 82.5 & 83.73 & 98.68 \\
\hline Error rate $(\%)$ & 1.3 & 2.3 & 83 & 0.56 & 0.12 \\
\hline
\end{tabular}

a Krill Herd optimization

Table 9 Evaluation of state-of-art approaches

\begin{tabular}{|c|c|c|c|c|c|c|c|}
\hline Author & Methods & $\begin{array}{l}\text { Accuracy } \\
(\%)\end{array}$ & $\begin{array}{l}\text { Precision } \\
(\%)\end{array}$ & $\begin{array}{l}\text { Recall } \\
(\%)\end{array}$ & $\begin{array}{l}\text { Specificity } \\
(\%)\end{array}$ & $\begin{array}{l}\text { Sensitivity } \\
(\%)\end{array}$ & $\begin{array}{l}\text { Error } \\
\text { rate }(\%)\end{array}$ \\
\hline Zheng et al. [48] & DLA-EABA & 97.2 & 95.4 & 96.95 & 96.5 & 98.3 & 2.8 \\
\hline Zhang et al. [47] & $\mathrm{CNN}$ & 81 & 79 & 75 & 82 & 79 & 19 \\
\hline Zhang et al. [47] & CLSTM & 90 & 86 & 87 & 91 & 89 & 10 \\
\hline Vaka et al. [40] & DNNS & 97.21 & 97.9 & 97.01 & 96 & 96.5 & 2.79 \\
\hline $\begin{array}{l}\text { Melekoodappattu and } \\
\text { Subbian [23] }\end{array}$ & ELM-FOA & 99.04 & 100 & 97.5 & 98 & 97.5 & 0.96 \\
\hline Piantadosi et al. [29] & Deep CNN & 99.16 & 98.56 & 98.34 & 99.49 & 96.85 & 0.84 \\
\hline $\begin{array}{l}\text { Ghasemzadeh et al. } \\
\text { [9] }\end{array}$ & $\begin{array}{l}\text { ML based } \\
\text { GWT }^{a}\end{array}$ & 93.9 & 92.4 & 91 & 92 & 95.1 & 6.1 \\
\hline $\begin{array}{l}\text { Yavuz and Eyupoglu } \\
\text { [45] }\end{array}$ & GRNN & 97.73 & 100 & 99.88 & 98.23 & 97.86 & 2.27 \\
\hline Shen et al. [36] & DL & 97 & 96 & 95.6 & 80.1 & 86.1 & 3 \\
\hline Proposed work & $\begin{array}{r}\text { BPBRW with } \\
\text { HKH-ABO }\end{array}$ & 99.6 & 100 & 99.9 & 95.78 & 98.68 & 0.12 \\
\hline
\end{tabular}

a Gabor wavelet transform

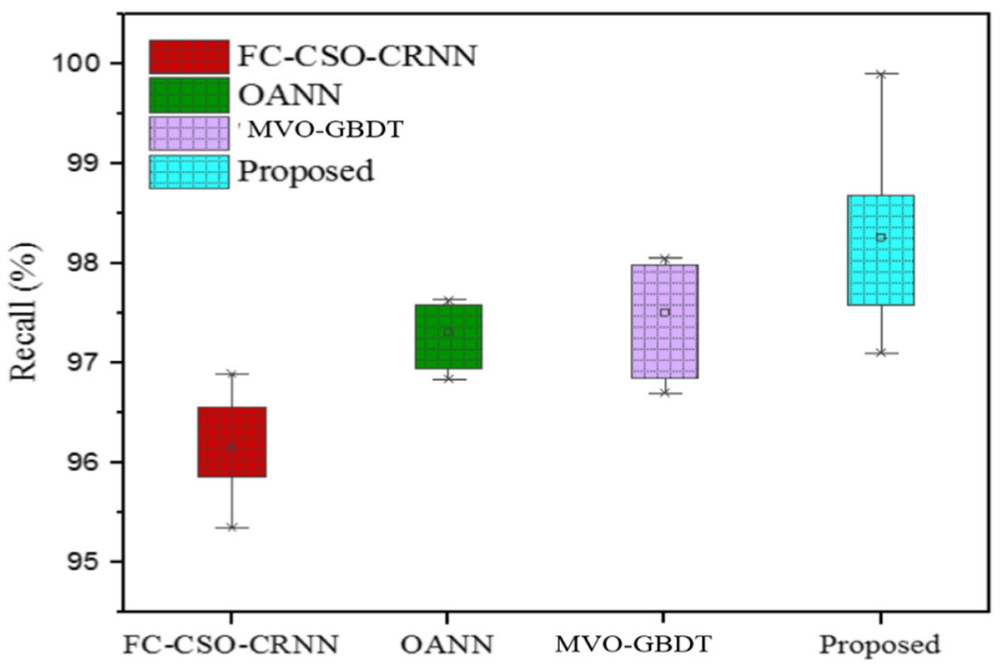

Fig. 10 Comparison of recall 


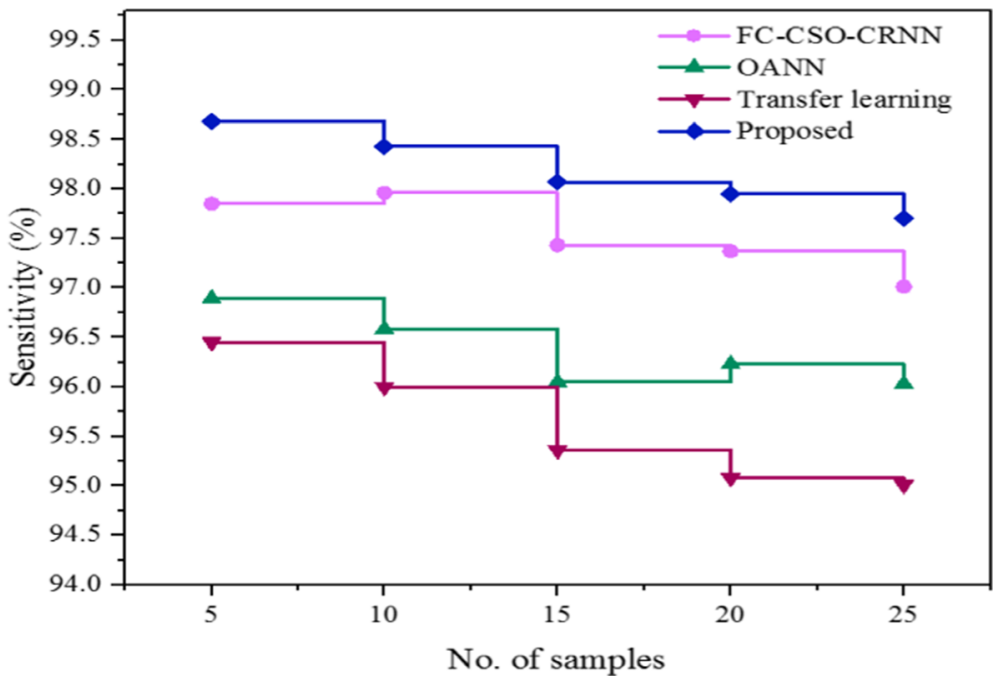

Fig. 11 Comparison of sensitivity

the proposed hybrid model has attained better results. This is the motivation for this proposed HKH-ABO model.

Here, the error rate is mentioned by analyzing the average of all test samples. Also, the error rate is calculated by validating the overall function of the proposed model. But the accuracy is validated based on the disease prediction. Thus, the accuracy is gained as $99.6 \%$, even the error rate is $0.12 \%$.

Hence, the developed BPBRW with HKH-ABO model has predicted breast cancer in an early stage with high accuracy rate, sensitivity, precision, Recall, specificity, and lower error rate, evaluation of state-of-art approaches are shown in Table 9, here comparing all those existing model the proposed model has attempted the better results in all parameters.

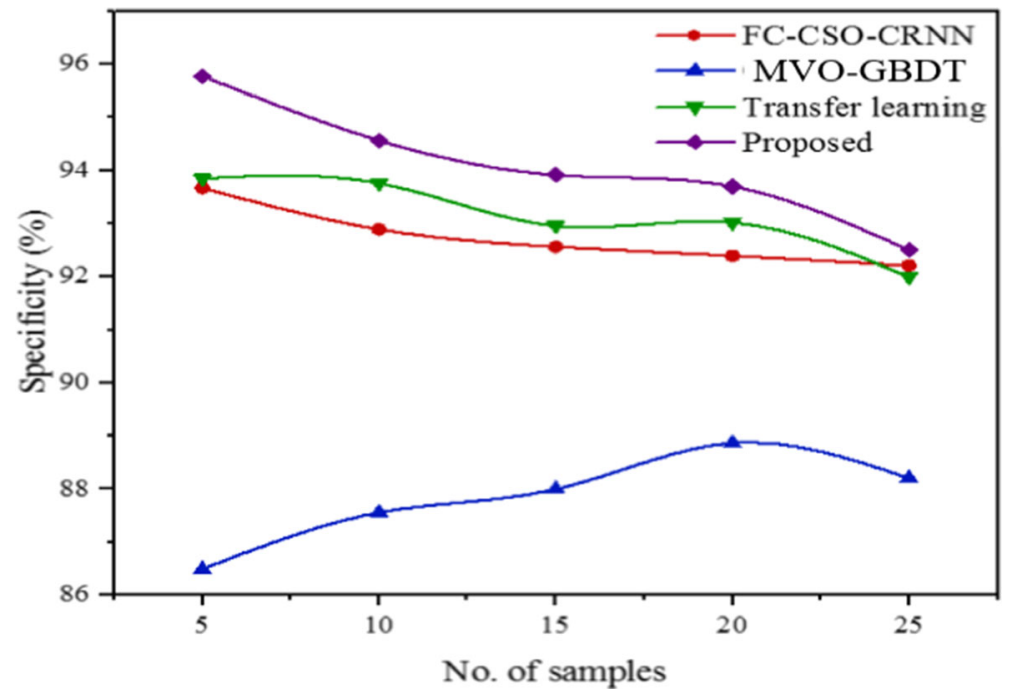

Fig. 12 Comparison of specificity 


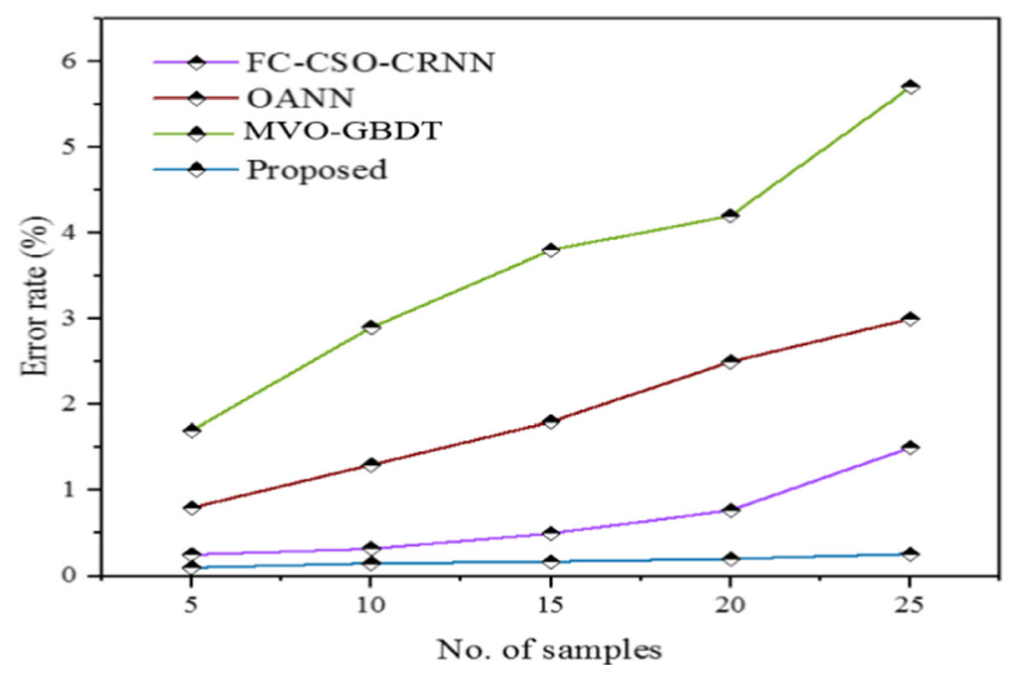

Fig. 13 Comparison of error rate

The performance of the proposed BPBRW with the HKH-ABO model is compared with which are mentioned in Table 10. Therefore, the proposed approach attained higher efficiency for detecting breast cancer than other techniques.

\subsection{Discussion}

To find the merits, demerits and future direction, state of the art was tabulated here in Table 10. Several existing works such as DLA-EABA [48], CLSTM [47], Conventional CNN [47], DNNS [40], ELM-FOA [23], GRNN [45], KNN and SVM [33], taxonomy model [2], F-CI [18],CNN[16], autoencoder CNN [39], FC-CSO-CRNN [27], OANN [38], MVO-GBDT [13], GWT [9], DL [44] and risk score model [5] are discussed in this section. However, several techniques have been met difficulties in disease classification steps because of complex data. Furthermore, some approaches have recorded design complexity and consumed more energy and time. Hence, this paper has designed a novel framework with deep hybrid learning and optimization approaches to resolving all issues. Also, to reduce data complexity, the filter model was implemented to remove the training error in the preprocessing model.

Hence, the proposed approach is named as BPBRW with HKH-ABO. In addition, the time complexity of the proposed model is $3 \mathrm{~s}$ for one run. But the only drawback in this model is that it has taken a few more times to design this hybrid model. So in the future, optimizing the design parameters will enhance the performance by minimizing the designing time. Hence, the proposed approach is applicable in clinical application by approving this mechanism from any medical professionals. This approach is efficient to process in a MRI based breast cancer detection sector.

\section{Conclusion}

A novel BPBRW with HKH-ABO approach has developed in the python environment to detect breast cancer in an earlier stage. Initially, the collected MRI breast image dataset is 
Table 10 Summary of state-of-art techniques

\begin{tabular}{|c|c|c|c|}
\hline Author & Method & Pros & Cons \\
\hline $\begin{array}{l}\text { Sadhukhan et al. } \\
\text { [33] }\end{array}$ & KNN and SVM & $\begin{array}{l}\text { It classified the affected part cell } \\
\text { nuclei and predicts the tumor } \\
\text { growth in early stage. }\end{array}$ & $\begin{array}{l}\text { This classification takes more time } \\
\text { period. }\end{array}$ \\
\hline $\begin{array}{l}\text { Benhammou et al. } \\
\text { [2] }\end{array}$ & $\begin{array}{c}\text { Taxonomy } \\
\text { model }\end{array}$ & $\begin{array}{l}\text { Easily classify and predict the } \\
\text { disease. }\end{array}$ & $\begin{array}{l}\text { Prediction accuracy of this model } \\
\text { is not efficient. }\end{array}$ \\
\hline Kumar et al. [18] & F-CI & $\begin{array}{l}\text { It attained better classification } \\
\text { accuracy. }\end{array}$ & $\begin{array}{l}\text { The interior process of this model } \\
\text { is difficult. }\end{array}$ \\
\hline Khan et al. [16] & $\mathrm{CNN}$ & $\begin{array}{l}\text { It effectively identified the breast } \\
\text { cancer tissues. }\end{array}$ & $\begin{array}{l}\text { It can utilize a large time period for } \\
\text { the identification of the affected } \\
\text { part. }\end{array}$ \\
\hline Toğaçar et al. [39] & $\begin{array}{l}\text { CNN with an } \\
\text { auto-encoder } \\
\text { model }\end{array}$ & This model detects the breast tumor. & $\begin{array}{l}\text { The overall performance of this } \\
\text { model is low. }\end{array}$ \\
\hline $\begin{array}{l}\text { Patil and Biradar } \\
\quad \text { [27] }\end{array}$ & FC-CSO-CRNN & $\begin{array}{l}\text { it has gained better accuracy for } \\
\text { predicting the affected region }\end{array}$ & $\begin{array}{l}\text { The designing process has taken } \\
\text { more time }\end{array}$ \\
\hline $\begin{array}{l}\text { Supriya and } \\
\text { Deepa [38] }\end{array}$ & OANN & $\begin{array}{l}\text { It has utilized less energy to run the } \\
\text { process }\end{array}$ & $\begin{array}{l}\text { However, it has required less } \\
\text { energy to run the process }\end{array}$ \\
\hline Junior et al. [13] & MVO-GBDT & Less error rate was recorded & It has consumed more energy. \\
\hline Zheng et al. [48] & DLA-EABA & It has gained $96.5 \%$ specificity & However, it is expensive in cost \\
\hline Zhang et al. [47] & $\mathrm{CNN}$ & $\begin{array}{l}\text { It has taken less duration to execute } \\
\text { the process }\end{array}$ & It has gained less accuracy \\
\hline Vaka et al. [40] & DNNS & $\begin{array}{l}\text { It has gained the finest accuracy for } \\
\text { disease classification }\end{array}$ & Time complexity \\
\hline $\begin{array}{l}\text { Melekoodappattu } \\
\text { and Subbian } \\
{[23]}\end{array}$ & ELM-FOA & $\begin{array}{l}\text { This approach has gained } 100 \% \\
\text { precision }\end{array}$ & complexity in design \\
\hline $\begin{array}{l}\text { Piantadosi et al. } \\
\text { [29] }\end{array}$ & DNN & $\begin{array}{l}\text { It has gained better accuracy for all } \\
\text { types of dataset }\end{array}$ & Time complexity \\
\hline $\begin{array}{l}\text { Ghasemzadeh } \\
\text { et al. [9] }\end{array}$ & GWT & It has gained average accuracy & $\begin{array}{l}\text { if the data is complex it has } \\
\text { reported high error rate }\end{array}$ \\
\hline $\begin{array}{l}\text { Yavuz and } \\
\text { Eyupoglu [45] }\end{array}$ & GRNN & $\begin{array}{l}\text { It has achieved better accuracy for } \\
\text { identifying the affected region }\end{array}$ & $\begin{array}{l}\text { It has needed more resources to } \\
\text { design the model }\end{array}$ \\
\hline Shen et al. [36] & $\begin{array}{l}\text { end to end } \\
\text { training DL } \\
\text { paradigm }\end{array}$ & $\begin{array}{l}\text { It has the capacity to train large } \\
\text { datasets }\end{array}$ & Time complexity \\
\hline Yala et al. [44] & DL & $\begin{array}{l}\text { The DL method is tested for both } \\
\text { mammogram and MRI images } \\
\text { and has gained better accuracy. }\end{array}$ & $\begin{array}{l}\text { However, it has reported high flaw } \\
\text { score }\end{array}$ \\
\hline $\begin{array}{l}\text { Dembrower et al. } \\
\text { [5] }\end{array}$ & risk score model & $\begin{array}{l}\text { Several risk factors are analyzed in a } \\
\text { detailed way }\end{array}$ & It has consumed more energy \\
\hline proposed & $\begin{array}{r}\text { BPBRW with } \\
\text { HKH-ABO }\end{array}$ & $\begin{array}{l}\text { It has attained the finest result } \\
\text { comparing all those techniques, } \\
\text { less error rate, less energy } \\
\text { consumption. Accurate severity } \\
\text { analysis of breast cancer in early } \\
\text { stage }\end{array}$ & $\begin{array}{l}\text { Because of designing two hybrid } \\
\text { models (deep learning and } \\
\text { optimization), it has taken few } \\
\text { more duration }\end{array}$ \\
\hline
\end{tabular}

trained to the system. Hereafter, to remove the training errors preprocess function has been performed. Consequently, the preprocessed data was imported to the classification layer then the feature extraction process and classification functions were done. Finally, the outcome of the proposed model has verified that it can effectively detect breast cancer in an early stage, such as benign type or malignant type. Additionally, the achievement of the projected scheme was evaluated with the prevailing schemes in terms of various metrics like accuracy, Recall, 
sensitivity, precision, and error rate. Hence, the projected mechanism has been achieved $99.6 \%$ higher accuracy with a $0.12 \%$ lower error rate; also, the time complexity of the proposed model is $3 \mathrm{~s}$, which shows the efficacy of the proposed model. In addition, the drawback of the proposed model is less security and designing time. In the future, implementing hybrid optimization with a security approach for tuning the design parameters will enhance the performance by avoiding adversarial attack and minimizing the designing time.

\section{Declarations}

Ethical approval All applicable institutional and/or national guidelines for the care and use of animals were followed.

Informed consent For this type of study formal consent is not required.

Conflict of interest The authors declare that they have no potential conflict of interest.

\section{References}

1. Bacolod MD, Huang J et al (2020) Prediction of blood-based biomarkers and subsequent design of bisulfite PCR-LDR-qPCR assay for breast cancer detection. BMC Cancer 20(1):85. https://doi.org/10.1186/s12885020-6574-4

2. Benhammou Y, Achchab B, Herrera F, Tabik S (2020) BreakHis based breast cancer automatic diagnosis using deep learning: taxonomy, survey and insights. Neurocomputing 375:9-24. https://doi.org/10.1016/j. neucom.2019.09.044

3. Chaudhary PK, Pachori RB (2021) FBSED based automatic diagnosis of COVID-19 using X-ray and CT images. Comput Biol Med 134:104454. https://doi.org/10.1016/j.compbiomed.2021.104454

4. Chaudhary PK, Pachori RB (2021) Automatic diagnosis of glaucoma using two-dimensional Fourier-Bessel series expansion based empirical wavelet transform. Biomed Signal Process Control 64:102237. https://doi. org/10.1016/j.bspc.2020.102237

5. Dembrower K, Liu Y, Azizpour H et al (2020) Comparison of a deep learning risk score and standard mammographic density score for breast cancer risk prediction. Radiology 294(2):265-272. https://doi.org/ 10.1148/radiol.2019190872

6. Dong Y, Liu Y, Chen J et al (2021) Comparison of postoperative CT-and preoperative MRI-based breast tumor bed contours in prone position for radiotherapy after breast-conserving surgery. Eur Radiol 31(1): 345-355. https://doi.org/10.1007/s00330-020-07085-0

7. dos Santos JCM, Carrijo GA et al (2020) Fundus image quality enhancement for blood vessel detection via a neural network using CLAHE and wiener filter. Res Biomed Eng:1-13. https://doi.org/10.1007/s42600020-00046-y

8. Du J, Vong CM, Chen CLP (2020) Novel efficient RNN and LSTM-like architectures: recurrent and gated broad learning systems and their applications for text classification. IEEE Trans Cybern 51(3):1586-1597. https://doi.org/10.1109/TCYB.2020.2969705

9. Ghasemzadeh A, Azad SS, Esmaeili E (2019) Breast cancer detection based on Gabor-wavelet transform and machine learning methods. Int J Mach Learn Cyber 10(7):1603-1612. https://doi.org/10.1007/s13042018-0837-2

10. Gupta V, Pachori RB (2021) FBDM based time-frequency representation for sleep stages classification using EEG signals. Biomed Signal Process Control 64:102265. https://doi.org/10.1016/j.bspc.2020.102265

11. Hu Q, Whitney HM, Giger ML (2020) A deep learning methodology for improved breast cancer diagnosis using multiparametric MRI. Sci Rep 10(1):1-11. https://doi.org/10.1038/s41598-020-67441-4

12. Jiang $X$ (2020) Isolated Chinese sign language recognition using gray-level co-occurrence matrix and parameter-optimized medium Gaussian support vector machine. Frontiers in Intelligent Computing: Theory and Applications, Springer, Singapore, pp 182-193. https://doi.org/10.1007/978-981-13-9920-6_19

13. Junior GB, da Rocha SV et al (2019) Breast cancer detection in mammography using spatial diversity, geostatistics, and concave geometry. Multimed Tools Appl 78(10):13005-13031. https://doi.org/10.1007/ s11042-018-6259-z 
14. Kesavan D, Periyathambi E, Chokkalingam A (2022) A proportional fair scheduling strategy using multiobjective gradient-based African buffalo optimization algorithm for effective resource allocation and interference minimization. Int J Commun Syst 35(1):e5003. https://doi.org/10.1002/dac.5003

15. Khalil R, Osman NM et al (2020) Unenhanced breast MRI: could it replace dynamic breast MRI in detecting and characterizing breast lesions? Egypt J Radiol Nucl Med 51(1):1-8. https://doi.org/10.1186/ s43055-019-0103-y

16. Khan SU, Islam $\mathrm{N}$ et al (2019) A novel deep learning based framework for the detection and classification of breast cancer using transfer learning. Pattern Recogn Lett 125:1-6. https://doi.org/10.1016/j.patrec.2019. 03.022

17. Kim J, Han BK, Ko EY et al (2022) Prediction of pathologic complete response on MRI in patients with breast cancer receiving neoadjuvant chemotherapy according to molecular subtypes. Eur Radiol:1-11. https://doi.org/10.1007/s00330-021-08461-0

18. Kumar M, Kulkarni AJ, Satapathy SC (2020) A hybridized data clustering for breast Cancer prognosis and risk exposure using fuzzy C-means and cohort intelligence. Optimization in Machine Learning and Applications. Springer, Singapore, pp 113-126. https://doi.org/10.1007/978-981-15-0994-0 7

19. Lee JY, Lee K, Seo BK, Cho KR et al (2022) Radiomic machine learning for predicting prognostic biomarkers and molecular subtypes of breast cancer using tumor heterogeneity and angiogenesis properties on MRI. Eur Radiol 32(1):650-660. https://doi.org/10.1007/s00330-021-08146-8

20. Li L, Pan X, Yang H et al (2020) Multi-task deep learning for fine-grained classification and grading in breast cancer histopathological images. Multimed Tools Appl 79(21):14509-14528. https://doi.org/10. 1007/s11042-018-6970-9

21. Ma M, Gan L, Liu Y, Jiang Y et al (2022) Radiomics features based on automatic segmented MRI images: prognostic biomarkers for triple-negative breast cancer treated with neoadjuvant chemotherapy. Eur J Radiol 146:110095. https://doi.org/10.1016/j.ejrad.2021.110095

22. Madhavan S, Tripathy RK, Pachori RB (2019) Time-frequency domain deep convolutional neural network for the classification of focal and non-focal EEG signals. IEEE Sensors J 20(6):3078-3086. https://doi.org/ 10.1109/JSEN.2019.2956072

23. Melekoodappattu JG, Subbian PS (2020) Automated breast cancer detection using hybrid extreme learning machine classifier. J Ambient Intell Humaniz Comput:1-10. https://oi.org/10.1007/s12652-020-02359-3

24. Meng Z, Huang H, Huang D, Zhang F, Mi P (2021) Functional metal-organic framework-based nanocarriers for accurate magnetic resonance imaging and effective eradication of breast tumor and lung metastasis. J Colloid Interface Sci 581:31-43. https://doi.org/10.1016/j.jcis.2020.07.072

25. Mohsenpourian M, Asharioun H, Mosharafian N (2021) Training fuzzy inference system-based classifiers with krill herd optimization. Knowl Based Syst 214:106625. https://doi.org/10.1016/j.knosys.2020.106625

26. Nayak SR, Nayak DR, Sinha U et al (2021) Application of deep learning techniques for detection of COVID-19 cases using chest X-ray images: a comprehensive study. Biomed Signal Process Control 64: 102365. https://doi.org/10.1016/j.bspc.2020.102365

27. Patil RS, Biradar N (2020) Automated mammogram breast cancer detection using the optimized combination of convolutional and recurrent neural network. Evolutionary Intelligence:1-16. https://doi.org/10.1007/ s12065-020-00403-X

28. Peng C, Zhang Y, Zheng J et al (2022) IMIIN: an inter-modality information interaction network for 3D multi-modal breast tumor segmentation. Comput Med Imaging Graph 95:102021. https://doi.org/10.1016/j. compmedimag.2021.102021

29. Piantadosi G, Sansone M et al (2020) Multi-planar 3D breast segmentation in MRI via deep convolutional neural networks. Artif Intell Med 103:101781. https://doi.org/10.1016/j.artmed.2019.101781

30. Qiao M, Suo S, Cheng F et al (2021) Three-dimensional breast tumor segmentation on DCE-MRI with a multilabel attention-guided joint-phase-learning network. Comput Med Imaging Graph 90:101909. https:// doi.org/10.1016/j.compmedimag.2021.101909

31. Qiao M, Liu C, Li Z et al (2022) Breast tumor classification based on MRI-US images by disentangling modality features. IEEE J Biomed Health Inform. https://doi.org/10.1109/JBHI.2022.3140236

32. Ravi V, Alazab M, Srinivasan S, Arunachalam A, Soman KP (2021) Adversarial defense: DGA-based botnets and DNS homographs detection through integrated deep learning. IEEE Trans Eng Manag:1-11. https://doi.org/10.1109/TEM.2021.3059664

33. Sadhukhan S, Upadhyay N, Chakraborty P (2020) Breast Cancer diagnosis using image processing and machine learning. Emerging Technology in Modelling and Graphics. Springer, Singapore, pp 113-127. https://doi.org/10.1007/978-981-13-7403-6_12

34. Saranyaraj D, Manikandan M, Maheswari S (2020) A deep convolutional neural network for the early detection of breast carcinoma with respect to hyper-parameter tuning. Multimed Tools Appl 79(15):1101311038. https://doi.org/10.1007/s11042-018-6560-x 
35. Sheela CJJ, Suganthi G (2020) An efficient denoising of impulse noise from MRI using adaptive switching modified decision based unsymmetric trimmed median filter. Biomed Signal Process Control 55:101657. https://doi.org/10.1016/j.bspc.2019.101657

36. Shen L, Margolies LR et al (2019) Deep learning to improve breast cancer detection on screening mammography. Sci Rep 9(1):1-12. https://doi.org/10.1038/s41598-019-48995-4

37. Sriram S, Vinayakumar R, Alazab M, Soman KP (2020) Network flow based IoT botnet attack detection using deep learning. In: IEEE INFOCOM 2020-IEEE conference on computer communications workshops (INFOCOM WKSHPS). IEEE, pp 189-194. https://doi.org/10.1109/INFOCOMWKSHPS50562.2020. 9162668

38. Supriya M, Deepa AJ (2019) A novel approach for breast cancer prediction using optimized ANN classifier based on big data environment. Health Care Manag Sci:1-13. https://doi.org/10.1007/s10729-019-09498-w

39. Toğaçar M, Ergen B, Cömert Z (2020) Application of breast cancer diagnosis based on a combination of convolutional neural networks, ridge regression and linear discriminant analysis using invasive breast cancer images processed with autoencoders. Med Hypotheses 135:109503. https://doi.org/10.1016/j.mehy.2019. 109503

40. Vaka AR, Soni B, Reddy S (2020) Breast cancer detection by leveraging machine learning. ICT Express. https://doi.org/10.1016/j.icte.2020.04.009

41. Vidal J, Vilanova JC, Martí R (2022) A U-net ensemble for breast lesion segmentation in DCE MRI. Comput Biol Med 140:105093. https://doi.org/10.1016/j.compbiomed.2021.105093

42. Vinayakumar R, Alazab M, Srinivasan S, Pham QV, Padannayil SK, Simran K (2020) A visualized botnet detection system based deep learning for the internet of things networks of smart cities. IEEE Trans Ind Appl 56(4):4436-4456. https://doi.org/10.1109/TIA.2020.2971952

43. Yadav SS, Jadhav SM (2020) Thermal infrared imaging based breast cancer diagnosis using machine learning techniques. Multimed Tools Appl:1-19. https://doi.org/10.1007/s11042-020-09600-3

44. Yala A, Lehman C, Schuster T, Portnoi T, Barzila R (2019) A deep learning mammography-based model for improved breast cancer risk prediction. Radiology 292(1):60-66. https://doi.org/10.1148/radiol. 2019182716

45. Yavuz E, Eyupoglu C (2020) An effective approach for breast cancer diagnosis based on routine blood analysis features. Med Biol Eng Comput 58:1583-1601. https://doi.org/10.1007/s11517-020-02187-9

46. Yu X, Chen H, Liang M, Xu Q, He L (2020) A transfer learning-based novel fusion convolutional neural network for breast cancer histology classification. Multimed Tools Appl:1-15. https://doi.org/10.1007/ s11042-020-09977-1

47. Zhang Y, Chen JH, Lin Y et al (2020) Prediction of breast cancer molecular subtypes on DCE-MRI using convolutional neural network with transfer learning between two centers. Eur Radiol:1-9. https://doi.org/10. 1007/s00330-020-07274-x

48. Zheng J, Lin D, Gao Z, Wang S, He M, Fan J (2020) Deep learning assisted efficient AdaBoost algorithm for breast Cancer detection and early diagnosis. IEEE Access. https://doi.org/10.1109/ACCESS.2020. 2993536

Publisher's note Springer Nature remains neutral with regard to jurisdictional claims in published maps and institutional affiliations. 